\title{
Scan Strategies for Electron Energy Loss Spectroscopy at Optical and Vibrational Energies in Perylene Diimide Nanobelts
}

\author{
Sean M. Collins ${ }^{1 *}$, Demie M. Kepaptsoglou ${ }^{2}$, Duncan N. Johnstone ${ }^{1}$, Tom Willhammar ${ }^{3}$, Raj Pandya ${ }^{4}$, \\ Jeffrey Gorman ${ }^{4}$, Richard Friend ${ }^{4}$, Akshay Rao ${ }^{4}$, Paul A. Midgley ${ }^{1}$, Quentin Ramasse ${ }^{5}$ \\ 1. Department of Materials Science and Metallurgy, University of Cambridge, Cambridge, UK. \\ 2. SuperSTEM, Daresbury, UK and Department of Physics, University of York, York, UK. \\ 3. Department of Materials and Environmental Chemistry, Stockholm University, Stockholm, Sweden. \\ 4. Department of Physics, University of Cambridge, Cambridge, UK. \\ 5. SuperSTEM, Daresbury, UK and School of Chemical and Process Engineering and School of Physics, \\ University of Leeds, Leeds, UK. \\ * Corresponding author: smc204@cam.ac.uk
}

Organic materials hold significant promise for scalable and processable optoelectronics from solar energy applications to light emitting diodes (LEDs). The molecular building blocks of many organic crystalline semiconductors, moreover, provide a route to functionalization and derivatization, enabling tunable photophysics such as modification of absorption, fluorescence, and charge mobility through modifications in the intramolecular bonding and the molecular packing in the crystal. The optical properties in organic semiconductors arise from the excitonic states defined by the molecule and the resulting intermolecular interactions in the solid state often dominated by $\pi-\pi$ stacking in common aromatic molecules. Perylene diimide (PDI) represents a major class of n-type organic semiconductors which exhibits long-range exciton diffusion in quasi-one dimensional nanostructures $[1,2]$.

Measurement of the nanoscale electronic structure in PDI nanobelts is a key objective for identifying factors that reduce exciton lifetime such as variation in energy levels and structural defects, critical for improving optoelectronic device performance. PDI materials are relatively stable under thermal treatment and optical illumination [1], but nevertheless organic semiconductors are often highly sensitive to electron beam damage [3,4]. The interaction between molecular vibrations and the electronic structure (the vibronic structure) is also a major factor in determining exciton lifetimes [2, 3], motivating spatially resolved electron energy loss spectroscopy (EELS) measurements with a focused nanoscale electron probe and with meV energy resolution in the scanning electron microscope (STEM). Two PDI derivatives were prepared in nanobelt form and characterized using a Hermes UltraSTEM $100 \mathrm{MC}$ (Nion) operated at $60 \mathrm{kV}$. The general structure of PDI is presented in Figure 1(a). The terminal R-group was a cyclohexyl group $\left(-\mathrm{C}_{6} \mathrm{H}_{11}\right)$ or a branched alkyl 'swallow tail' chain $\left(-\mathrm{CH}\left(\mathrm{CH}_{2} \mathrm{CH}_{2} \mathrm{CH}_{3}\right)_{2}\right)$. The crystal structures of both derivatives were determined by rotation electron diffraction. Figure 1(b)(c) shows the structure for the cyclohexyl derivative. Figure 1(d) presents an annular dark field micrograph of a section of a PDI nanobelt. The prevailing $\pi-\pi$ stacking direction is indicated along the long axis of the nanobelt.

A variety of scan strategies were explored to determine suitable dose and signal to noise characteristics for acquiring EELS from PDI nanobelts at optical and vibrational energies. For measurements of the optical states (1-10 eV), the energy resolution was set to approximately $25 \mathrm{meV}$ (full width at half maximum of the zero loss peak). For measurements of vibrational states $(<0.5 \mathrm{eV})$, the energy resolution was set to approximately $15 \mathrm{meV}$. Scans across the width of the nanobelts were found to result in significant changes in the EEL spectra after just one exposure (Figure 2(a)), notably reduced intensity of 
the $\mathrm{C}-\mathrm{H}$ stretch $(0.4 \mathrm{eV})$ and reduced intensity at $2-3 \mathrm{eV}$ and at $6 \mathrm{eV}$. The spectra shown are the result of 10 spectra summed from the aloof portion up to and including the first penetrating trajectory in the nanobelt (2.6 $\mathrm{nm}$ pixels). Line profiles in near-aloof geometry (at the edge of the nanobelt), however, enabled recording spectra continuously over long distances without showing signs of damage. Figure 2(b)-(c) depicts a sum spectrum over $500 \mathrm{~nm}$ along a nanobelt with an improved signal-to-noise relative to a single local-area scan as in Figure 2(a). These strategies also enabled vibrational EELS showing a pronounced resolved peak at $1700-1800 \mathrm{~cm}^{-1}$ likely attributed to the $\mathrm{C}=\mathrm{O}$ functional groups. These spatially resolved EEL spectra from PDI now report on the electronic structure of individual wires on length scales inaccessible by conventional optical techniques. Additionally, the capability for scanning along the crucial exciton diffusion direction establishes a route to map changes in electronic and vibrational structure at structural defects in these materials systems [5].

\section{References:}

[1] Y Che et al., Chemistry of Materials 21 (2009), p. 2930.

[2] D Chaudhuri et al., Nano Letters 11 (2010), p. 488.

[3] A Eggeman et al., Nature Materials 12 (2013), p. 1045.

[4] J Alexander et al., Journal of Materials Chemistry A 4 (2016), p. 13636.

[5] SMC gratefully acknowledges support from the Microanalysis Society Goldstein Scholar Award and the Henslow Research Fellowship at Girton College, Cambridge. P.A.M. acknowledges support from the EPSRC (EP/R025517). SuperSTEM is the UK National Research Facility for Advanced Electron Microscopy, supported by EPSRC. The research was also supported by the European Research Council (291522-3DIMAGE and 670405-EXMOLS).

(a)

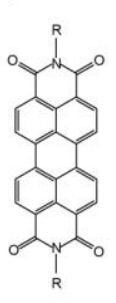

(b)

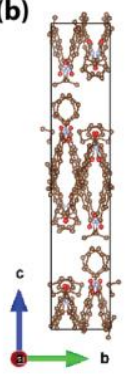

(c)

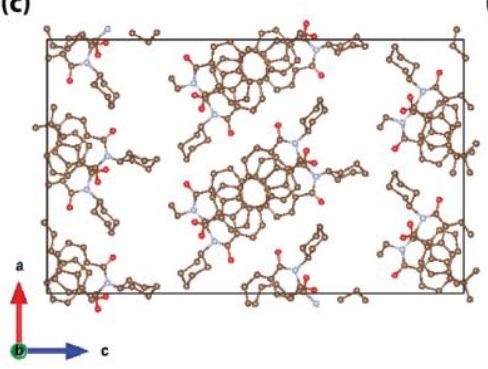

(d)

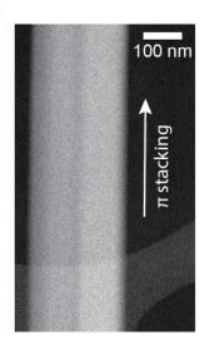

Figure 1. (a) Molecular structure of PDI and (b)-(c) crystal structure determined by rotation electron diffraction for a PDI derivative $(\mathrm{R}=$ cyclohexyl). The hydrogen atoms are not shown. (d) ADF-STEM micrograph of a nanobelt.

(a)

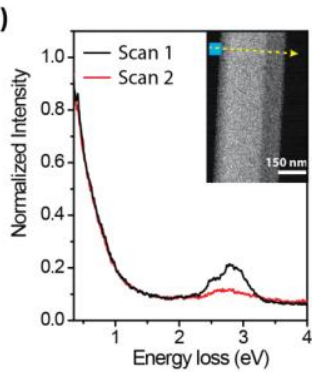

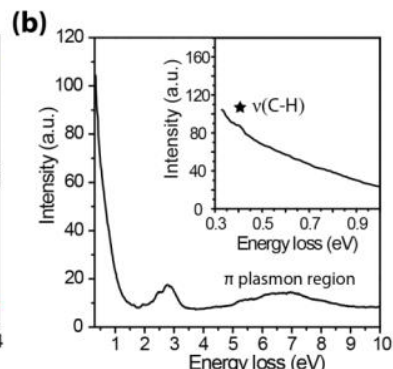

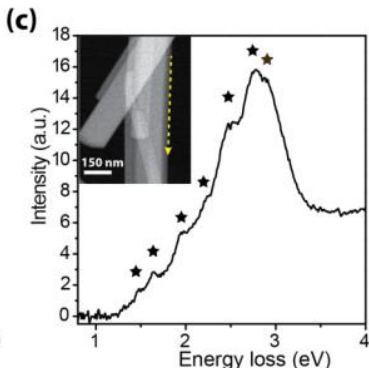

Figure 2. (a) Changes in low loss EELS observed after repeated scanning. The inset shows the total scan (dashed arrow) and the position for the spectra (square). (b)-(c) A low loss EELS sum spectrum recorded from a scan along the edge of a belt. In (c), the line scan position (inset) is shown alongside the background subtracted sum spectrum (background model: Lorentzian function with offset). 